\title{
Downlink Throughput Maximization in CDMA Wireless Networks*
}

\author{
M. Kemal Karakayali Roy Yates Leonid V. Razoumov \\ Department of Electrical and Computer Engineering \\ Wireless Information Networks Laboratory (WINLAB) \\ Rutgers University, 73 Brett Road Piscataway, NJ 08854-8060 \\ e-mail: kemal,ryates,leor@winlab.rutgers.edu
}

June 27, 2004

\begin{abstract}
We investigate optimum rate assignment scheme maximizing network throughput on the downlink of a multirate CDMA wireless network. Systems employing orthogonal variable spreading factor (OVSF) codes as well as systems employing multiple codes have been studied. Our objective is to maximize the network throughput under constraints on total transmit power, total bandwidth and individual QoS requirements specified in terms of minimum rates. First, users are ordered based on their transmit energy per bit requirements to achieve the target received energy per bit to interference power spectral density ratio at the receivers. Based on the initial ordering, we prove that for systems employing multiple codes, greedy rate scheduling yields maximum network throughput. For systems employing variable spreading codes, we show that greedy rate scheduling is optimal if the minimum rate requirement of a user is larger than or equal to the minimum rate requirement of any other user with a larger transmit energy per bit requirement. Simulation results verify the superiority of the greedy algorithm under various system and channel assumptions.
\end{abstract}

\section{Introduction}

Wireless data networks provide access to multimedia applications such as video streaming and internet, together with classical applications such as voice. While there is an increasingly high demand for wireless services, radio resources are often scarce, and therefore a careful and efficient allocation of limited resources is vital. The challenge is that different applications have different quality of service requirements. For example, while downloading a music file may be delayed a few minutes or more, real time services such as voice are delay intolerant,

*This paper was presented, in part, at the IEEE Wireless Communications and Networking Conference (WCNC), New Orleans, LA., March 2003. This work is supported in part by the NJ Commission on Science and Technology under the NJCWT program. 
and require a minimum service rate. In this study, we consider minimum service rate as a quality of service metric, and investigate how to maximize the network throughput in a multirate CDMA downlink.

For the CDMA uplink, resource allocation problems are studied extensively in [1-6]. The common objective in these studies is either to maximize the sum of rates (or utility), or to minimize the total transmit power, subject to constraints on individual transmit power assignments, total bandwidth, the quality of reception in terms of SINR targets, minimum service requirement of each user (fairness), or delay. For the CDMA downlink, resource allocation problems are studied in [7-14]. A hierarchical SIR and rate control algorithm is proposed in [7]. First, the mobiles determine their SIR targets using mobile specific information, and then the base station determines rate assignments using the limited feedback from the mobiles. In this paper, our analysis will answer how the base station algorithm in the hierarchical structure should work. Downlink scheduling for delay tolerant data services is considered in [8-11]. It is shown in [8] that a time division scheme in which users transmit one by one within each cell provides energy efficiency and increased capacity. Similarly, in [9], it is proven that, for data-only networks, the optimal scheme is to transmit to only one data user at a time, which is the technique used in the HDR system $[10,15]$. While these schemes attempt to guarantee long term nonzero average throughput to each user, they are not suitable if users have instantaneous service requirements, as in the case of voice, or realtime multimedia. In this paper, we define the instantaneous minimum rate as a quality of service metric, and analyze the optimal rate assignment scheme in this case. Our results coincide with the one user at a time conclusion [8-11], when there is no minimum service rate requirement.

A simplifying approach in resource optimization problems is to assume continuous rate and power assignments, instead of practical discrete system parameters, at the expense of an approximate solution. Here, we assume discrete rates corresponding to systems employing 
either multiple codes or variable spreading codes. In this case, the throughput maximization becomes a discrete optimization problem. We show that for systems employing multiple codes, the problem can be solved efficiently by a simple greedy algorithm with polynomial complexity. For systems employing variable spreading codes, the problem is more complicated and the greedy algorithm is optimal if the minimum rate requirements are ordered by the channel qualities.

The organization of the paper is as follows. The system model is described in Section 2. The problem statement is given in Section 3. In Sections 4, the rate assignment algorithms are introduced, and the optimality analysis are provided. Section 5 is on examples and simulation results for CDMA networks. We conclude the paper in Section 6.

\section{System Model}

Multiple Access Scheme: In CDMA wireless networks, multiple users share a common communication medium by means of spreading codes. There are two ways to assign spreading codes in such systems. First, in the third generation W-CDMA standard [16], multirate data service is provided by assigning each user a single spreading code with variable length. In such a scheme, spreading codes are obtained from a binary tree structure (Figure 1) and are called Orthogonal Variable Spreading Factor (OVSF) codes [17]. On the other hand, in multicode CDMA systems, each user can be provided with multiple spreading codes of fixed length, depending on the users' rate requests.

Figure 1 shows how OVSF codes are obtained from a binary tree structure. In the figure, $C_{i, j}$ represents $j$ th node on layer $i$ on the binary tree, and it corresponds to a unique spreading code (signature sequence) of length $2^{i}$ and rate $R_{0} / 2^{i}$ where $R_{0}$ is the root rate corresponding to the node $C_{0,1}$. Moreover, orthogonality of the assigned spreading codes is guaranteed by the fact that none of the parent-child node pairs is assigned at the same time. As an example, the nodes $C_{1,2}$ and $C_{2,4}$ in Figure 1 cannot be in use at the same time since 
$C_{1,2}$ is a prefix of $C_{2,4}$. Therefore, the assigned signature codes must have the prefix-free property.

Accordingly the prefix-free property imposes a constraint on the set of spreading codes available in OVSF CDMA systems. It is a well-known fact that the Kraft inequality determines whether a set of codes with specified lengths can be placed on the binary tree as a prefix-free set [18]. Denoting the length of the branch from the root node $\left(C_{0,1}\right.$ in Figure 1$)$ to the $u$ th user's node by $l_{u}$ and number of users by $N$, the Kraft Inequality,

$$
\sum_{u=1}^{N} 2^{-l_{u}} \leq 1
$$

must be satisfied to obtain a prefix-free set.

In the context of CDMA spreading codes, the Kraft inequality is a bandwidth constraint. Since $R_{0}$ denotes the root rate and represents the maximum total rate or the bandwidth of the system, it follows that the rate of user $u$ is

$$
R_{u}=\frac{R_{0}}{2^{l_{u}}}
$$

Multiplying both sides of (1) by $R_{0}$, the Kraft inequality becomes

$$
\sum_{u=1}^{N} R_{u} \leq R_{0}
$$

which states that $R_{0}$ is an upper bound on the throughput of an OVSF CDMA system. It is trivial to generalize the constraint (3) to the multicode CDMA case and $R_{0}$ represents the system bandwidth in this case.

Channel Model: We consider a single cell CDMA downlink with $N$ mobiles. Each mobile has a different application running, and therefore each has a specific QoS requirement in terms of a minimum service rate. For each transmitted bit of user $u$, there is a target received energy per bit to interference power spectral density ratio, $(E / I)_{u}^{r} \geq \gamma_{u}$, with which the receiver can decode the transmitted bits with an acceptable bit error rate (BER). 
The channel between the base station and each mobile can be modeled either as a frequency flat single path channel, or as a frequency selective multipath channel. These two models have quite different implications on the problem definition. Unlike the uplink where random spreading codes are used, orthogonal spreading codes are used on the downlink. In frequency flat channels, the orthogonality of the transmitted waveforms is preserved at the receiver. On the other hand, in a frequency selective multipath channel, the orthogonality is lost at the receivers. Our problem formulation will be general enough to account for both channel models. For each channel model, we determine the transmit energy per bit requirements to achieve the target SINR per bit $\gamma_{u}$ at the receivers.

In a frequency flat channel, the orthogonality implies that the overall system can be modeled as a number of parallel channels. Since there is no interference across parallel channels, the system only compensates for the background noise, i.e. $(E / I)_{u}^{r}=\left(E / N_{0}\right)_{u}^{r} \geq$ $\gamma_{u}$, and therefore user $u$ requires received energy per bit $E_{u}^{r} \geq \gamma_{u} N_{0}$ where $N_{0}$ is the noise power spectral density. In this case, the transmit energy per bit should satisfy $E_{u}^{t}=E_{u}^{r} / h_{u} \geq$ $\gamma_{u} N_{0} / h_{u}$, where $h_{u}$ denotes the link gain for user $u$.

When there is multipath in the channel, delayed versions of orthogonal spreading codes arrive at each receiver, leading to multi-access interference due to the loss of orthogonality between spreading codes. In the CDMA downlink, the loss of orthogonality can be characterized by the orthogonality factor $(\mathrm{OF})$, which is defined as the fraction of received downlink power converted by multipath into multi-access interference $[19,20]$. As noted in $[19,20]$, the orthogonality factor is a time-varying parameter that depends on the instantaneous multipath channels as well as the receiver structure and the spreading codes employed. On the other hand, in the analysis of the downlink, time average value of the orthogonality factor is traditionally employed $[19,20]$. This is due to the fact that data symbols, which are first spread by orthogonal codes, are then multiplexed and scrambled by cell-specific random long codes before transmission. In this case, the analysis is more tractable if the interference is 
modeled using its average over the length of the random long code. Here we follow the same convention, and assume that user $u$ has an average orthogonality factor of $\bar{\beta}_{u}$. In this case, we can express $(E / I)_{u}^{r}$ as

$$
\left(\frac{E}{I}\right)_{u}^{r}=\frac{W}{R_{u}} \frac{p_{u} h_{u}}{\left(\bar{\beta}_{u} \sum_{i \neq u} p_{i} h_{u}+N_{0} W\right)}=\frac{W}{R_{u}} \frac{p_{u} h_{u}}{\left(\bar{\beta}_{u} h_{u} \sum_{i} p_{i}-\bar{\beta}_{u} p_{u} h_{u}+N_{0} W\right)} \geq \gamma_{u}
$$

where $W$ denotes the spreading bandwidth, $R_{u}$ denotes the rate assignment, and $p_{u}$ is the transmit power. In deriving (4), we inherently assume rake reception where the gain sum of all paths is represented by the path loss $h_{u}$.

Here we are interested in a practical power limited system where the power constraint is a bottleneck on system throughput. In this case, it is expected that the base station uses any nonzero residual power to increase the throughput with some nonzero rate (or for some users to achieve higher received SINR per bit and better reception quality). The base station therefore transmits at its peak power. This is the case in [7] and [14] where the rate assignments are not restricted to be discrete, and it is shown that either the power constraint is achieved with equality, i.e $\sum_{i} p_{i}=P$, or the whole bandwidth is allocated. Our design in this paper will be a conservative one in which the received SINR is guaranteed to exceed $\gamma_{u}$ given $\sum_{i} p_{i} \leq P$. By upperbounding $\sum_{i} p_{i}$ by $P$ and using (4), we require that

$$
\left(\frac{E}{I}\right)_{u}^{r} \geq \frac{W}{R_{u}} \frac{p_{u} h_{u}}{\left(\bar{\beta}_{u} h_{u} P-\bar{\beta}_{u} p_{u} h_{u}+N_{0} W\right)} \stackrel{(a)}{\geq} \gamma_{u}
$$

Thus, the set of rate and power assignments satisfying (a) will satisfy the inequality (4) as well. Rewriting $(a)$, it follows that

$$
p_{u} h_{u} \geq \frac{\frac{R_{u}}{W}\left(\bar{\beta}_{u} h_{u} P+N_{0} W\right) \gamma_{u}}{1+\frac{R_{u} \gamma_{u} \bar{\beta}_{u}}{W}}
$$

Using (6) and the fact that the transmit power is $p_{u}=E_{u}^{t} R_{u}$, we obtain

$$
E_{u}^{t}=\frac{p_{u}}{R_{u}} \geq \frac{\left(\bar{\beta}_{u} h_{u} P+N_{0} W\right) \gamma_{u}}{\left(W+R_{u} \bar{\beta}_{u} \gamma_{u}\right) h_{u}}
$$

Notice that when $\bar{\beta}_{u}=0,(7)$ reduces to $E_{u}^{t} \geq \gamma_{u} N_{0} / h_{u}$, which is the SINR constraint in flat channels. We note that, in the multicode CDMA case, $E_{u}^{t}$ is required for one of the 
multicodes (we will denote its rate by $R_{s}$ in the rest of the paper). Assignment of multiple codes to a user generates self interference. In this case, each spreading code needs to be treated separately, and $E_{u}^{t}$ has to be calculated for each as if every other spreading code is an interferer signal.

\section{Problem Statement}

For both multicode CDMA systems and OVSF CDMA systems, the throughput maximization can be formulated as the following discrete optimization problem over the vector $\eta=\left(\eta_{1}, \ldots, \eta_{N}\right)$ of user rates:

$$
\begin{aligned}
\max _{\eta} & \sum_{u=1}^{N} \eta_{u} \leq R_{0} \\
\text { subject to } & \sum_{u=1}^{N} E_{u}^{t} \eta_{u} \leq P \\
& \eta_{u} \geq \eta_{\min , u} \quad u=1, \ldots, N \\
& \eta_{u} \in S=\left\{\eta^{1}, \eta^{2}, \ldots, \eta^{M}\right\}
\end{aligned}
$$

where each user selects a rate $\eta_{u}$ from the set $S=\left\{\eta^{1}, \eta^{2}, \ldots, \eta^{M}\right\}$ such that the sum rate is maximized within the bandwidth constraint (8). Note that (8a) is the constraint on the total transmit power, and each bit of user $u$ is transmitted with energy $E_{u}^{t}$ so as to satisfy the target received SINR $\gamma_{u}$, i.e. to satisfy (7) with equality. Moreover, (8b) represents the users' individual bandwidth constraints.

For multicode CDMA systems, $\eta_{u}=R_{s} n_{u}$ where $R_{s}$ denotes the rate corresponding to a single spreading code, and $n_{u}$ denotes the number of spreading code assignments. Thus, the set of possible rate assignments are given by $S=\left\{R_{s}, 2 R_{s}, 3 R_{s}, \ldots, M R_{s}\right\}$, where $M$ is the total number of multicodes.

For OVSF CDMA systems, $\eta_{u}=R_{0} 2^{-l_{u}}$ where $R_{0}$ denotes the root rate on the binary code tree, and $l_{u}$ denotes the length of the branch from the root node $\left(C_{0,1}\right.$ in Figure 1) to the $u$ th user's node. Thus, the set of possible rate assignments are given by 
$S=\left\{R_{0}, R_{0} / 2, R_{0} / 4, \ldots\right\}$. On the other hand, each user has a minimum rate requirement $\eta_{\min , u}=R_{0} / 2^{L_{u}}$ where $L_{u}$ is the minimum depth on the binary code tree below which spreading codes cannot provide the user's minimum rate.

\section{Algorithms and Analysis}

For systems using OVSF codes, the rate assignment problem is equivalent to determining the relative distances $l_{u}$ of spreading codes to the root node on the binary code tree. In terms of the vector $\mathbf{l}=\left(l_{1}, \ldots, l_{N}\right)$ of distances, the problem (8) becomes

$$
\begin{aligned}
\max _{\mathbf{l}} & \sum_{u=1}^{N} R_{0} 2^{-l_{u}} \\
\text { subject to } & \sum_{u=1}^{N} E_{u}^{t} R_{0} 2^{-l_{u}} \leq P \\
& \sum_{u=1}^{N} 2^{-l_{u}} \leq 1 \\
& l_{u} \in\left\{0,1, \ldots, L_{u}\right\}
\end{aligned}
$$

where the bandwidth constraint in (8) is written in terms of the Kraft inequality (9b), and the minimum rate constraint $(8 \mathrm{~b})$ is embedded in the set of possible distances $(9 \mathrm{c})$.

The objective function (9) is a nonlinear function of integer branch length $l_{u}$. Moreover, the energy per bit $E_{u}^{t}$ depends on the rate assignment (7), and therefore is a function of the branch length $l_{u}$, which complicates the problem further. The problem above is a mixedinteger nonlinear program (MINLP), which is generally hard to solve, and requires exhaustive search over the set of possible rate combinations because MINLP problems combine the difficulties of their subclasses: the combinatorial nature of mixed integer programs, and the difficulty in solving nonlinear programs, both of which are among the class of NP-complete problems. Fortunately, we realize that under certain conditions on the constraint set, and under realistic system assumptions, the problem can be solved by a simple algorithm with polynomial complexity. First, an instance of interest is when $E_{i}^{t}<E_{j}^{t}$ implies $L_{i} \leq L_{j}$ 
for all $i$ and $j$, i.e. when the minimum rate requirement of a user is larger than or equal to the minimum rate requirement of any other user with a larger transmit energy per bit requirement. Second, we are interested in the cases where the transmit energy per bit $E_{u}^{t}$ does not depend on the rate assignment $R_{u}$. This is clearly the case in flat channels where $\bar{\beta}_{u}=0$ in (7). On the other hand, in frequency selective channels, we make the large system assumption, and notice that in a system with a large number of users, each having nonzero minimum rate requirements, it is unlikely to have users dominating the whole available bandwidth by themselves, i.e $W \gg R_{u}=R_{0} 2^{-l_{u}}$ in general. For example in a WCDMA downlink, the spreading bandwidth is $W=3.84 \times 10^{6}$ chips/second while the minimum spreading length is 4 chips, corresponding to the root rate of $R_{0}=0.96 \times 10^{6} \mathrm{bits} / \mathrm{second}$. Even in a very lightly loaded system, say there are only 2 users, the user with the better channel can receive at most half of the root rate (since 2 users can only be placed somewhere below the root node on the binary code tree), which is $0.48 \times 10^{6} \mathrm{bits} /$ second, and the ratio $W / R_{u}$ is 8 . This ratio will increase geometrically down the binary code tree and can be as large as 512, which many users will have in a highly loaded system. In this case, it can be shown that the dependence of $E_{u}^{t}$ on $R_{u}$ is not significant. Notice that a tighter constraint on $E_{u}^{t}$ can be written as

$$
E_{u}^{t} \stackrel{(a)}{\geq} \frac{\left(\bar{\beta}_{u} h_{u} P+N_{0} W\right) \gamma_{u}}{W h_{u}} \stackrel{(b)}{\geq} \frac{\left(\bar{\beta}_{u} h_{u} P+N_{0} W\right) \gamma_{u}}{\left(W+R_{u} \bar{\beta}_{u} \gamma_{u}\right) h_{u}}
$$

where $(a)$ and $(b)$ implies (7). On the other hand, for typical values of $\bar{\beta}_{u} \approx 0.3[20], \gamma_{u}=5$ $(\approx 7 \mathrm{~dB})$ and under the assumption $W \gg R_{u},(b)$ can be approximated by an equality ${ }^{1}$, and therefore the constraint on $E_{u}^{t}$ does not depend significantly on $R_{u}$. While this analysis is quite accurate for very low data rates, users with relatively high rates achieve larger SINR per bit when the constraint $(a)$ is satisfied.

\footnotetext{
${ }^{1} \mathrm{~A}$ similar assumption $[7]$ that in a system with a large number of users with nonzero minimum rates, transmit power for a single user is much less than the total power constraint, i.e. $P=\sum_{i} p_{i} \gg p_{u}$, leads to the same result.
} 


\subsection{Rate Assignment Algorithm in OVSF CDMA Systems}

The rate assignment algorithm is given in Figure 2. First, the minimum rate requirement of each user is provided. The rest of the algorithm is greedy in nature, and the objective is to increase (to double in the binary tree case) the rate of the user who requires minimum energy per bit. Based on the initial ordering by transmit energy per bit $E_{u}^{t}$ requirements, the algorithm attempts to maximize the rate of a user at each greedy step within the total transmit power constraint (9a) and the bandwidth constraint (9b) expressed in terms of the Kraft inequality.

Notice that spreading code of user $u$ resides on layer $l_{u}$ of the binary code tree in Figure 1. Although $l_{u}$ uniquely determines the rate assignment (2), it does not tell us which node on layer $l_{u}$ of the code tree should be assigned to user $u$. On the other hand, satisfying the Kraft Inequality (1) guarantees the fact there is at least a set of $N$ spreading codes on the binary code tree such that spreading code of user $u$ is placed on layer $l_{u}$, the rates of all other users are not affected by this placement (although spreading codes might shift on the same layer) and all spreading codes in the set are mutually orthogonal as a result of the prefix free property. The shifts or replacements of spreading codes on the same layer on the binary code tree are implementation issues and such shifts do not affect the assigned rate of a user. Thus, the way the spreading code replacements or shifts occurs at each step of the algorithm, the subject of $[21,22]$, is not addressed in this study.

\subsection{Greedy Optimality in OVSF CDMA Systems}

The basic idea is that the set of rate assignments by any existing optimal algorithm can be made more "greedy" by reordering and reassigning user rates in a way to favor the users with better channels, and all these reassignments are feasible on the binary code tree. Moreover, as the rate assignments by the optimal algorithm look more like the greedy assignments, the total power is reduced while keeping the total throughput constant. Note that if $R_{i}<R_{j}$ and 
$E_{i}^{t}<E_{j}^{t}$ for any two users $i$ and $j$, we can swap the rates without changing the total sum of rates as long as the new assignments do not violate the minimum rate constraints. Denoting the total transmit power before and after swapping $R_{i}$ and $R_{j}$ by $P$ and $P^{s}$ respectively, it follows that

$$
P-P^{s}=\left[E_{i}^{t} R_{i}+E_{j}^{t} R_{j}\right]-\left[E_{i}^{t} R_{j}+E_{j}^{t} R_{i}\right]=\left(R_{j}-R_{i}\right)\left[E_{j}^{t}-E_{i}^{t}\right]>0
$$

where the last inequality follows from the fact that $E_{j}^{t}>E_{i}^{t}$ and $R_{j}>R_{i}$.

We first prove the optimality of the greedy algorithm in the two user case. We then generalize the proof to any number of users.

Theorem 1. Given that the user with smaller transmit energy per bit requirement has also larger or equal minimum rate requirement, the greedy algorithm solves the OVSF rate assignment problem for $N=2$ users.

Proof. A general form of the problem in the 2 user case is as follows

$$
\begin{aligned}
\max & R_{0}\left(2^{-l_{1}}+2^{-l_{2}}\right) \\
\text { subject to } & E_{1}^{t} R_{0} 2^{-l_{1}}+E_{2}^{t} R_{0} 2^{-l_{2}} \leq P^{\prime} \\
& 2^{-l_{1}}+2^{-l_{2}} \leq \rho \\
& l_{i} \in\left\{0,1, \ldots, L_{i}\right\}, \quad i=1,2
\end{aligned}
$$

for any $0<P^{\prime} \leq P$ and $0<\rho \leq 1$. Without loss of generality, assume the first user requires smaller transmit energy per bit $E_{1}^{t}<E_{2}^{t}$.

Consider an optimal vector $\mathbf{l}^{*}=\left[l_{1}^{*}, l_{2}^{*}\right]$ yielding maximum throughput. Given $L_{1} \leq L_{2}$, we can always choose the optimal vector $\mathbf{l}^{*}$ in such a way that $l_{1}^{*} \leq l_{2}^{*}$; otherwise if $l_{1}^{*}>l_{2}^{*}$, user 1 and user 2 can exchange the assigned values so that the total throughput remains the same and the new assignments even save some power. Note that such an exchange does not violate individual minimum rate constraints because $L_{1} \leq L_{2}$. Our greedy algorithm yields 
the vector $\hat{\mathbf{l}}=\left[\hat{l}_{1}, \hat{l}_{2}\right]$, such that

$$
\begin{aligned}
& \hat{l}_{1}=\min \left\{l \in £ \mid 2^{-l}+2^{-L_{2}} \leq \rho, \quad E_{1}^{t} R_{0} 2^{-l}+E_{2}^{t} R_{0} 2^{-L_{2}} \leq P^{\prime}\right\}, \\
& \hat{l}_{2}=\min \left\{l \in £ \mid 2^{-l}+2^{-\hat{l}_{1}} \leq \rho, \quad E_{2}^{t} R_{0} 2^{-l}+E_{1}^{t} R_{0} 2^{-\hat{l}_{1}} \leq P^{\prime}\right\} .
\end{aligned}
$$

We compare $\mathbf{l}^{*}$ and $\hat{\mathbf{l}}$,

- Assume $l_{1}^{*}<\hat{l}_{1}$ : For user 1 , the greedy algorithm finds the smallest $\hat{l}_{1}$ on the binary tree, while power and bandwidth constraints are satisfied and the second user's spreading code resides on layer $L_{2}$. Thus $l_{1}^{*}<\hat{l}_{1}$ is impossible; otherwise $\hat{l}_{1}$ would not be the local minimum choice.

- Assume $l_{1}^{*}>\hat{l}_{1}$ : In this case the smallest possible value of $l_{1}^{*}$ is $\hat{l}_{1}+1$. Since $l_{1}^{*} \leq l_{2}^{*}$, we have $\hat{l}_{1}+1 \leq l_{1}^{*} \leq l_{2}^{*}$. It follows that

$$
R_{0}\left(2^{-l_{1}^{*}}+2^{-l_{2}^{*}}\right) \leq R_{0}\left(2^{-\left(\hat{l}_{1}+1\right)}+2^{-\left(\hat{l}_{1}+1\right)}\right)=R_{0} 2^{-\hat{l}_{1}}<R_{0}\left(2^{-\hat{l}_{1}}+2^{-\hat{l}_{2}}\right)
$$

since $0 \leq \hat{l}_{2} \leq L_{2}$. Equation (15) asserts that the rate achieved by $\mathbf{l}^{*}$ is strictly less than the rate achieved by $\hat{\mathbf{l}}$, which contradicts the optimality of $\mathbf{l}^{*}$ implying that $l_{1}^{*}>\hat{l}_{1}$ is also impossible. As a result we conclude that $l_{1}^{*}=\hat{l}_{1}$.

Similarly, the greedy algorithm makes a local minimum choice for user 2 while the first user's spreading code resides on layer $\hat{l}_{1}$. Because $l_{1}^{*}=\hat{l}_{1}$, and $\hat{l}_{2}$ is a local minimum, $\hat{l}_{2} \leq l_{2}^{*}$ must be true. On the other hand if $\hat{l}_{2}<l_{2}^{*}$, then the optimal $\mathbf{l}^{*}$ offers smaller throughput compared to the greedy $\hat{\mathbf{l}}$, which is a contradiction. Therefore $l_{2}^{*}=\hat{l}_{2}$ must be true.

Theorem 2. Given that the minimum rate requirement of a user is larger than or equal to the minimum rate requirement of any other user with a larger transmit energy per bit requirement, the greedy algorithm solves the OVSF rate assignment problem.

Proof. Here we will prove a more general statement that the greedy algorithm solves rate maximization problem for any power constraint $0<P^{\prime} \leq P$ and any bandwidth constraint 
$0<\rho \leq 1$, corresponding to a "partial" binary code tree. The general form of the problem is

$$
\begin{aligned}
\max & \sum_{u=1}^{N} R_{0} 2^{-l_{u}} \\
\text { subject to } & \sum_{u=1}^{N} E_{u}^{t} R_{0} 2^{-l_{u}} \leq P^{\prime} \leq P \\
& \sum_{u=1}^{N} 2^{-l_{u}} \leq \rho \leq 1 \\
& l_{u} \in\left\{0,1, \ldots, L_{u}\right\}
\end{aligned}
$$

Without loss of generality, we can assume that $E_{1}^{t}<E_{2}^{t}<\cdots<E_{N}^{t}$. Consider an optimal vector $\mathbf{l}^{*}=\left[l_{1}^{*}, l_{2}^{*}, \ldots, l_{N}^{*}\right]$ yielding maximum total throughput. Given $L_{1} \leq L_{2} \leq$ $\cdots \leq L_{N}$, any feasible set of optimal rate assignments can be reordered in such a way that $l_{1}^{*} \leq l_{2}^{*} \leq \cdots \leq l_{N}^{*}$, without violating individual minimum rate constraints. It is important to notice that, given $E_{i}^{t}<E_{j}^{t}$ and $l_{i}^{*}>l_{j}^{*}$, an exchange between assignments of users $i$ and $j$ does not violate minimum rate constraints if $L_{i} \leq L_{j}$. In addition, such an exchange always saves power (11); therefore the power constraint is not violated while the throughput remains constant.

Our greedy algorithm yields the vector $\hat{\mathbf{l}}=\left[\hat{l}_{1}, \hat{l}_{2}, \ldots, \hat{l}_{N}\right]$. The proof goes by induction. We already proved the greedy optimality for the two user case in Theorem 1 . Here, we assume that Theorem 2 is true for any system of $\tilde{N}<N$ users. We also assume that there is a feasible rate assignment vector for the problem (16). Therefore, optimal and greedy solutions are both feasible and we will consider them below.

Definition 1. Let $A$ be a user index such that $\hat{l}_{u}=l_{u}^{*}$ for $u=1, \ldots, A-1$, and $\hat{l}_{A} \neq l_{A}^{*}$.

Due to local optimality of the greedy algorithm, we have $\hat{l}_{A} \leq l_{A}^{*}-1$. First, we suppose $A>1$. In this case at least the first user gets the same rate assignment by both algorithms (greedy and optimal). The remaining $N-1$ users can be assigned in a greedy fashion because our induction hypothesis stipulates that for any $\tilde{N}<N$ users, greedy assignments 
are optimal. This proves Theorem 2 for the case of $A>1$. Thus, it remains to consider the case of $A=1$. Since optimal $\mathbf{l}^{*}$ achieves maximum total throughput, employing $\hat{l}_{A} \leq l_{A}^{*}-1$ for the case $A=1$, we can write that

$$
2^{-l_{1}^{*}}+\sum_{u=2}^{N} 2^{-l_{u}^{*}} \geq 2^{-\hat{l}_{1}}+\sum_{u=2}^{N} 2^{-\hat{l}_{u}} \geq 2^{-\left(l_{1}^{*}-1\right)}+\sum_{u=2}^{N} 2^{-L_{u}}
$$

Equation (17) asserts there is a new set of rate assignments on the binary code tree in which the rate of user 1 is doubled from the optimal $2^{-l_{1}^{*}}$ to $2^{-\left(l_{1}^{*}-1\right)}$, while the rest of the users get their minimum rates; clearly the throughput of the new assignments does not exceed the optimal throughput of $\mathbf{l}^{*}$. The question is whether the new assignments of users $2, \ldots, N$ can be further increased on the binary code tree until achieving the optimal throughput of $\mathbf{l}^{*}$, and without violating the power constraint.

Lemma 1. For any $\mathbf{l}^{*}=\left[l_{1}^{*}, l_{2}^{*}, \ldots, l_{N}^{*}\right]$ satisfying (17), we can always find a set of assignments $\tilde{l}_{2}^{*}, \ldots, \tilde{l}_{N}^{*}$ such that

$$
\begin{aligned}
2^{-l_{1}^{*}}+\sum_{u=2}^{N} 2^{-l_{u}^{*}} & =2^{-\left(l_{1}^{*}-1\right)}+\sum_{u=2}^{N} 2^{-\tilde{l}_{u}^{*}} \\
l_{u}^{*} & \leq \tilde{l}_{u}^{*} \leq L_{u}, u=2, \ldots, N
\end{aligned}
$$

Proof of Lemma 1. Here we give a constructive proof of Lemma 1 by providing an explicit algorithm, Figure 3, which computes the new assignments $\tilde{l}_{2}^{*}, \ldots, \tilde{l}_{N}^{*}$. The algorithm starts with $\mathbf{l}^{*}$, and halves the rate of a chosen user (releases half of the user's bandwidth) at each iteration $n$. We denote the amount of bandwidth released at $n$th iteration by $\triangle_{n}$, and the aggregate bandwidth released up to the $n$th iteration by $J_{n}$. The basic idea is to show that eventually there will be enough bandwidth released to double the rate of user 1, i.e. $J_{n}$ equals to $2^{-l_{1}^{*}}$ at some point, and that the algorithm falls through Step 3 and always terminates.

Let's assume that there is no $n$ such that $J_{n}=2^{-l_{1}^{*}}$. Together with (17) it means that there will be such an iteration $t$ for which

$$
J_{t-1}=\sum_{n=1}^{t-1} \triangle_{n}<2^{-l_{1}^{*}}, \quad J_{t}=\sum_{n=1}^{t} \triangle_{n}>2^{-l_{1}^{*}} .
$$


We also observe that

$$
2^{-l_{1}^{*}} \geq \triangle_{1} \geq \triangle_{2} \geq \cdots \geq \triangle_{t}
$$

Partial ordering in (21) follows from Step 1 of the algorithm which always chooses the largest rate user to release its bandwidth. Due to the ordering in (21) and the definition of $t$, the difference between $J_{t}$ and $2^{-l_{1}^{*}}$ is smaller that the contribution from the last iteration

$$
J_{t}-2^{-l_{1}^{*}}=\sum_{n=1}^{t} \triangle_{n}-2^{-l_{1}^{*}}<\triangle_{t}
$$

We emphasize here that by construction $\triangle_{n}$ is always some negative integer power of two. Therefore, (21) implies that the ratios $\triangle_{n} / \triangle_{t}$ are integers for $n=1, \ldots, t$. Next, we divide both sides of (22) by $\triangle_{t}$, yielding

$$
\left(\sum_{n=1}^{t} \frac{\triangle_{n}}{\triangle_{t}}\right)-\frac{2^{-l_{1}^{*}}}{\triangle_{t}}<1 .
$$

In the above inequality, the left side is a positive integer due to (20), while the right side is a fractional number less than one. Therefore (23) contains a contradiction, proving that Step 3 terminates the algorithm which results in the set of assignments $\tilde{l}_{2}^{*}, \ldots, \tilde{l}_{N}^{*}$ as in Lemma 1.

With the help of Lemma 1, we can immediately construct a new rate assignment vector $\tilde{\mathbf{l}}^{*}=\left[l_{1}^{*}-1, \tilde{l}_{2}^{*}, \ldots, \tilde{l}_{N}^{*}\right]$ yielding the same throughput as the optimal assignments $\mathbf{l}^{*}$, yet because of (19), it consumes less energy. Notice that $\tilde{\mathbf{l}}^{*}$ looks more "greedy" than $\mathbf{l}^{*}$, i.e. user 1 with the minimum transmit energy per bit requirement gets an enhanced rate while the other users get less than or equal to their rate assignments in $\mathbf{l}^{*}$ due to (19).

To complete the proof of Theorem 2 in the case of $A=1$, Lemma 1 can be applied to $\tilde{\mathbf{l}}^{*}$ as well, i.e. starting from the new set of optimal assignments $\tilde{\mathbf{l}}^{*}$, we can construct another optimal set with the first user receiving $l_{1}^{*}-2$ and all other users receive less than or equal to their assignments in $\tilde{\mathbf{I}}^{*}$, but more than their minimum requirements. We can continue in 
this fashion until the first user receives $\hat{l}_{1}$, the assignment by the greedy algorithm, in an optimal set of assignments. At this point, we already proved the greedy optimality, i.e. for $A>1$ using our induction hypothesis that greedy assignments are optimal for any $\tilde{N}<N$ users. The greedy optimality proof of Theorem 1 in the two user case is therefore generalized to any number of users by induction.

It is interesting to note that the optimality of the greedy algorithm in OVSF CDMA systems depends on the minimum rate constraints. When users with worse channels require larger minimum rates, the greedy algorithm may not be the optimal way to assign user rates. As a simple example, assume there are two users in the system; the first with $E_{1}^{t}=1$, and the second with $E_{2}^{t}=1.25$. The power constraint is 11 , and minimum rate requirements are $R_{1, \min }=1$ and $R_{2, \min }=4$. Total power to provide the minimum rates is $P_{\min }=$ $\sum_{u=1}^{2} E_{u}^{t} R_{u, \min }=6$. Since the first user requires smaller transmit energy per bit, the greedy algorithm would double the first user's initial rate assignment using the remaining power budget of $11-6=5$. In this case, the greedy algorithm could at most assign 4 units of rate to user one, which requires an additional 3 units of power. The remaining $11-6-3=2$ units of power would not be enough to double second user's initial assignment from 4 to 8 , therefore the greedy algorithm would conclude $R_{1}=4, R_{2}=4$ and a total throughput of 8 units of rate. On the other hand, optimal throughput is 9 units of rate which is achieved when $R_{1}=1$ and $R_{2}=8$.

\subsection{Rate Assignment Algorithm in Multicode CDMA Systems}

A simple polynomial time greedy algorithm, given in Figure 4, solves the rate assignment problem in multicode systems. The optimal algorithm favors the user with the smallest transmit energy per bit requirement. After the algorithm assigns minimum rates and allocates corresponding spreading codes to each user, only the rate of the user with the minimum energy per bit requirement is maximized using the remaining power budget. 
The intuition is that if a spreading code is to be assigned, it is better if it is assigned to the user who can receive it with the smallest power (the smallest contribution to the total power). On the other hand, unlike the uplink where the multiaccess interference at the receiver depends on the channels of all users and the individual power assignments, the multiaccess interference on the downlink is a function of the user's own channel and the power assignments of interferer spreading codes. Thus, a reduction in the power of any interferer signal is beneficial to all users no matter who the spreading code is assigned to.

Theorem 3. The greedy algorithm solves any instance of the multicode rate assignment problem (8).

Proof. The optimality proof is similar to the one in the OVSF case. We refer to [23] for further details.

\section{$5 \quad$ Examples and Simulations}

We apply the algorithms on the downlink of a single cell CDMA wireless network. The system assumptions are consistent with $3 \mathrm{G}$ system specifications $[16,17]$. Spreading gain is assumed to be in the range from a minimum of 4 up to 512, which corresponds to 8 levels on the binary code tree including the root node. The spreading bandwidth is $3.84 \mathrm{MHz}$, the maximum base station transmission power is 10 Watts, the receiver noise, $N_{o} W$, is $10^{-10}$ Watts, and the target $(E / I)_{u}^{r}=\gamma_{u}$ is $5(7 \mathrm{~dB})$ (different applications may have different $\gamma_{u}$ targets, the algorithms are applicable in such cases as well). The $x$ any $y$ coordinates of each mobile are selected uniformly on (0-2000m) in a square cell of $4 \mathrm{~km}^{2}$. The path loss $L_{p}$ at any given distance $d$ is given by [24]

$$
L_{p}=L_{0}+10 \epsilon \log _{10}\left(d / d_{0}\right)+S ; \quad d \geq d_{0}
$$

where $L_{0}$ is the decibel path loss at distance $d_{0}, \epsilon$ is the path loss exponent, and $S$ is the shadow fading variation. The numerical values are $d_{0}=100 \mathrm{~m}, L_{0}=78 \mathrm{~dB}, S$ is normal with 
0 mean and $\sigma=8 \mathrm{~dB}$, and $\epsilon=4$. The multipath characteristics, or the frequency selectivity, of the channels are represented by the orthogonality factor $\bar{\beta}$; for simplicity we assume that all users have the same average $\bar{\beta}$. The first experiment is conducted for $\bar{\beta}=0$, which corresponds to a flat channel, $\bar{\beta}=0.1, \bar{\beta}=0.2, \bar{\beta}=0.5$ and $\bar{\beta}=0.8$. For each mobile, we randomly choose a minimum rate corresponding to one of $\{128,256,512\}$ length spreading codes with equal probability. We assume fixed modulation and coding ${ }^{2}$.

The average throughput of the greedy algorithm in an OVSF CDMA system is compared to the average optimal throughput of a system using multiple codes. The average throughput values are obtained over 500 simulation runs for a given number of users. In each simulation run, a random set of user locations, a set of independent random shadow fades, and a set of minimum rate requirements are generated. In case minimum rate requirements are not feasible, i.e. if providing minimum rates to each user at the target $(E / I)_{u}^{r}=\gamma_{u}$ requires more power than $P$, a new set of minimum rates are generated by decreasing the minimum rate requirements of the users who require the largest transmit energy per bit.

Figure 5 compares average throughput results in multirate CDMA systems. In the figure, the solid lines represent the OVSF throughput determined by the greedy algorithm, and the dotted lines represent the optimal throughput of a multicode system, which is achieved by the greedy algorithm for any set of minimum rates. Since any set of rates offered by variable spreading factor codes can be realized by multiple codes (for example a spreading code of length 128 offers the same rate as 4 spreading codes of length 512), the optimal throughput of a multicode system can be thought of as an upperbound to the average throughput of an OVSF system. The figure shows that the greedy OVSF throughput is very close to the optimal greedy multicode throughput, especially for large number of users. Moreover, we

\footnotetext{
${ }^{2}$ There is no explicit assumption on modulation and coding format. The only assumption is that they are the same and fixed for all users, which implies that spreading codes with the same lengths correspond to the same rate, and the rate assignments are proportional to the number of spreading code assignments in multicode systems, and are determined by the lengths of the spreading codes in variable spreading systems. In numerical calculations, we assumed uncoded QPSK, while the algorithms and analysis are valid for any fixed coding and modulation format.
} 
observe that the performance gap between multicode throughput and OVSF throughput closes as the number of users increases and the holes on the binary code tree get filled more efficiently by more users. Notice that it is much easier to manage orthogonal spreading codes in a multicode CDMA system; for example in a 2 user OVSF system, the user with a better channel can achieve at most half of the bandwidth (the node below the root node), while in a multicode CDMA system the same user can have the whole available bandwidth as long as the power constraint permits. Also note that, for large values of $\beta$, there is a decline in total throughput as the number of users increases. This is counter-intuitive since we expect the optimal scheme to exploit the multiuser diversity, i.e. users with favorable channel conditions, when the number of users increases. On the other hand, since each user requires a nonzero minimum rate, a large increase in the number of users results in larger interference floor for the system, and thus reduces the system capacity. This fact suggests that we employ a call admission scheme which limits the number admitted real-time users (i.e. users with minimum service rate requirements) to a certain number $(6-7$ users in Figure 5), instead of a call admission scheme which admits as many users as possible based on the feasibility of the minimum rates.

In Table 1 and Table 2, we present two sample cases in order to understand how various system parameters affect the rate assignment policy. In Table 1, individual rate assignments and corresponding path loss values are presented for a sample case of 10 users in a system using OVSF codes. Notice that except path loss, all other factors affecting the transmit energy per bit are the same for all users in the example. As a result, users with relatively small path loss values receive high throughput since they require lower transmit energy per bit. In the second example of Table 2, we assign rates to users who are close to the base station (or in a short range system) where the path loss is small, and the received multiaccess interference power may be much larger than the receiver noise, i.e. $\bar{\beta}_{u} h_{u} P \gg N_{0} W$. A simple link budget calculation shows that if the path loss is smaller than $50-60 \mathrm{~dB}$, the transmit 
energy per bit is mainly affected by the loss of orthogonality between spreading codes due to the multipath channel, and is independent of the path loss. For example, if $P=100 \mathrm{~mW}$, $\bar{\beta}_{u} \approx 0.1, N_{0} W \approx 10^{-10}$ Watts, and $h_{u}>10^{-6}$, then

$$
E_{u}^{t}=\frac{1}{h_{u}} \frac{\left(\bar{\beta}_{u} h_{u} P+N_{0} W\right) \gamma_{u}}{W+R_{u} \bar{\beta}_{u} \gamma_{u}} \approx \frac{\bar{\beta}_{u} P \gamma_{u}}{W+R_{u} \bar{\beta}_{u} \gamma_{u}}
$$

which is independent of $h_{u}$. In this case, the rate assignment policy is mainly affected by the orthogonality factor $\bar{\beta}_{u}$. We observe in Table 2 that the user with a moderate path loss (50 dB) but with the smallest orthogonality factor achieves the largest throughput.

\section{Conclusion}

In this paper, we investigated the throughput maximization problem on the downlink of CDMA wireless networks. For systems employing multiple codes, we derive the optimal greedy rate assignment scheme. For systems employing variable spreading codes, we derive and prove the conditions for the optimality of the greedy algorithm. Our simulation results show that the greedy algorithm, even when it is suboptimal in some instances of an OVSF system, is a good heuristic yielding close to optimal average throughput results in OVSF systems. As a future work, an interesting question is how to combine an admission control scheme with our rate assignment algorithm in a system with a dynamic traffic flow. In addition, one of our followup studies [25] investigates the effects of multiuser receivers, power control and spreading code selection on the downlink performance.

\section{Acknowledgements}

We would like to thank Dr. Larry Greenstein for his invaluable comments on the early draft.

\section{References}

[1] S. Ramakrishna and J.M. Holtzman. A Scheme for Throughput Maximization in a Dualclass CDMA System. IEEE Journal on Selected Areas in Communications, 16:830-844, 
August 1998.

[2] S. Ulukus and L. J. Greenstein. Throughput Maximization in CDMA Uplinks Using Adaptive Spreading and Power Control. In IEEE Symposium on Spread Spectrum Techniques and Applications, September 2000.

[3] S-J. Oh and K.M. Wasserman. Optimality of Greedy Power Control and Variable Spreading Gain in Multi-class CDMA Mobile Networks. In ACM/IEEE MobiCom, 1999.

[4] J. B. Kim and M.L Honig. Resource Allocation for Multiple Classes of DS-CDMA Traffic. IEEE Transactions on Vehicular Technology, 49:506-519, March 2000.

[5] F. Berggren and S.-L. Kim. Energy-Efficient Control of Rate and Power in DS-CDMA Systems. To appear in IEEE Transactions on Wireless Communications.

[6] A. Goldsmith S.A. Jafar. Adaptive Multirate CDMA for Uplink Throughput Maximization. IEEE Transactions on Wireless Communications, 2(2):218-228, March 2003.

[7] L. Song and N. Mandayam. Hierarchical SIR and Rate Control on the Forward Link for CDMA Data Users under Delay and Error Constraints. IEEE JSAC, 19(10):1871-1882, October 2001.

[8] F. Berggren, S.-L. Kim, R. Jntti, and J. Zander. Joint Power Control and Intra-cell Scheduling of DS-CDMA Non-real Time Data. IEEE Journal on Selected Areas in Communications, 19(10):1860-1870, 2001.

[9] A. Bedekar, Simon C. Borst, K. Ramanan, P. A. Whiting, and E. M. Yeh. Downlink Scheduling in CDMA Data Networks. Technical Report PNA-R9910, 31, 1999.

[10] Sem C. Borst and Phil Whiting. Dynamic Rate Control Algorithms for CDMA Throughput Optimization. In INFOCOM, pages 976-985, 2001. 
[11] M. Andrews, K. Kumaran, K. Ramanan, A. Stolyar, R. Vijayakumar, and P. Whiting. Providing Quality of Service over a Shared Wireless Link. IEEE Communications Magazine, February 2001.

[12] N. Joshi, S.R. Kadaba, S. Patel, and G.S. Sundaram. Downlink Scheduling in CDMA Data Networks. In ACM Mobicom 2000.

[13] C. Zhou, M.L Honig, S. Jordan, and R. Berry. Forward-link Resource Allocation for a Two-cell Voice Network with Multiple Service Classes. Wireless Communications and Networking, 2:1060-1065, March 2003.

[14] J. W. Lee, R. Mazumdar, and N. B. Shroff. Joint Power and Data Rate Allocation for the Downlink in Multi-class CDMA Wireless Networks. In The Fortieth Annual Allerton Conference on Communication, Control, and Computing, Monticello, IL, October 2002.

[15] P. Bender, P. Black, M. Grob, R. Padovani, N. Sindhushayana, and A. Viterbi. CDMA/HDR: A Bandwidth-efficient High-speed Wireless Data Service for Nomadic Users. IEEE Communications Magazine, pages 70-77, July 2000.

[16] F. Adachi, M. Sawahashi, and H. Suda. Wideband DS-CDMA for Next-Generation Mobile Communication Systems. IEEE Communications Magazine, 36(9):56-69, September 1998.

[17] F. Adachi, M. Sawahashi, and K. Okawa. Tree-structured Generation of Orthogonal Spreading Codes with Different Lengths for Forward Link of DS-CDMA Mobile Radio. IEEE Electronics Letters, 33(1):27-28, January 1997.

[18] T. Cover and J. Thomas. Elements of Information Theory. John Wiley Sons, 1991. 
[19] O. Awoniyi, N. Mehta, and L.J. Greenstein. Characterizing the Orthogonality Factor in WCDMA Downlinks. IEEE Transactions on Wireless Communications., 2(4), July 2003.

[20] N.B. Mehta, L.J. Greenstein, III T.M Willis, and Z. Kostic. Analysis Results for the Orthogonality Factor in WCDMA Downlinks. IEEE Transactions on Wireless Communications, 2002. to be published.

[21] T. Minn and K.-Y Siu. Dynamic Assignment of Orthogonal Variable Spreading Factor Codes in W-CDMA. IEEE J. Selected Areas Commun., 18(8):1429-1440, August 2000.

[22] Y-C. Tseng, C-M. Chao, and S-L Wu. Code Placement and Replacement Strategies for Wideband CDMA OVSF Code Tree Management. In Proc. of IEEE Globecom, volume 1, pages 562-566, 2001.

[23] M. Kemal Karakayali. Resource Allocation in Downlink Wireless Systems. Master's thesis, Department of Electrical and Computer Engineering, Rutgers University, October 2003.

[24] V. Erceg, L.J. Greenstein, S.Y. Tjandra, S.R. Parkoff, A. Gupta, B. Kulic, A.A. Julius, and R. Bianchi. An Empirically Based Path Loss Model for Wireless Channels in Suburban Environments. IEEE JSAC, 17(7), July 1999.

[25] M. K. Karakayali, R. D. Yates, and L. Razumov. Joint Power and Rate Control in Multiaccess Systems with Multirate Services. In CISS'03, The Johns Hopkins University, MD, March 2003. 


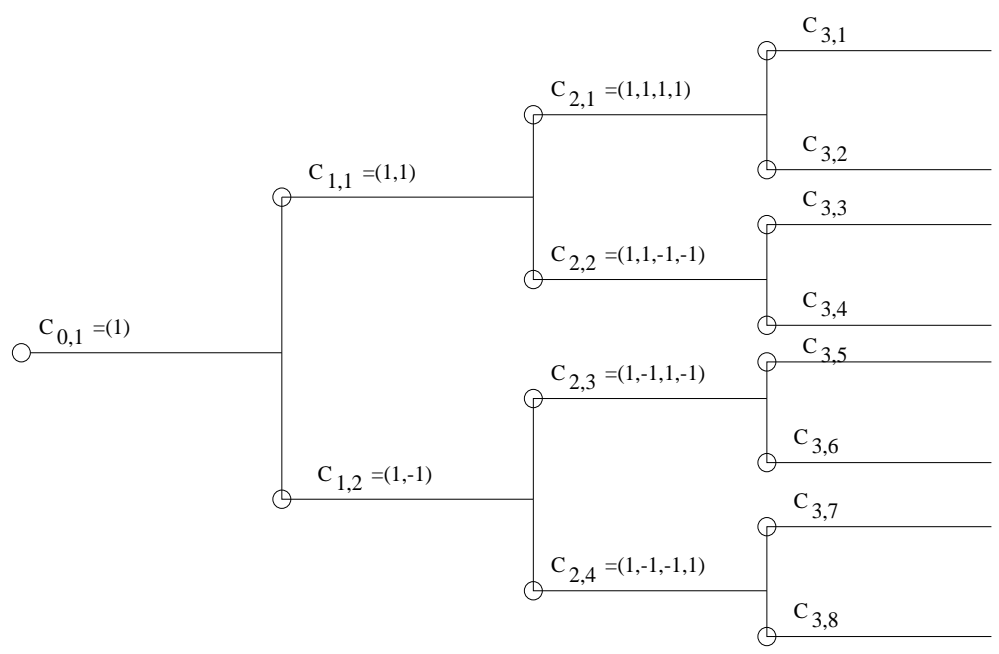

$\begin{array}{llcc}\text { layer } 0 & \text { layer } 1 & \text { layer } 2 & \text { layer } 3 \\ \mathrm{SF}=1 & \mathrm{SF}=2 & \mathrm{SF}=4 & \mathrm{SF}=8 \\ \text { Rate=R } & \text { Rate=R/2 } & \text { Rate }=\mathrm{R} / 4 & \text { Rate=R/8 }\end{array}$

Figure 1: OVSF Code Tree. $C_{i, j}$ represents node $j$ on layer $i$ and has a length of $\mathrm{SF}=2^{i}$

Input : $R_{0}, P, £_{u}=\left\{0,1, \ldots, L_{u}\right\}, \mathbf{E}_{b}^{t}=\left[E_{1}^{t}, E_{2}^{t}, \ldots, E_{N}^{t}\right]$ (in increasing order) Output : $\mathbf{l}=\left[l_{1}, \ldots, l_{N}\right]$

Initialization : for $u=1, \ldots, N, l_{u}=L_{u}$

$P_{t}=\sum_{u=1}^{N} E_{u}^{t} R_{0} 2^{-L_{u}}$

for $\mathbf{u}=1: \mathrm{N}$

$l_{u}=\min \left\{l \in £_{u} \mid 2^{-l}+\sum_{v \neq u} 2^{-l_{v}} \leq 1, \quad 2^{-l}-2^{-L_{u}} \leq \frac{P-P_{t}}{E_{u}^{t} R_{0}}\right\}$

$P_{t}=P_{t}+E_{u}^{t} R_{0}\left(2^{-l_{u}}-2^{-L_{u}}\right)$

end

Figure 2: Rate Assignment Algorithm for OVSF CDMA Systems

\begin{tabular}{|l|c|c|c|c|c|c|c|c|c|c|}
\hline User Index & 1 & 2 & 3 & 4 & 5 & 6 & 7 & 8 & 9 & 10 \\
\hline Path Loss $(\mathrm{dB})$ & 41 & 48 & 63 & 67 & 77 & 78 & 80 & 94 & 97 & 99 \\
\hline$R_{u, \min }(\mathrm{Kbps})$ & 15 & 60 & 15 & 15 & 15 & 15 & 15 & 60 & 15 & 60 \\
\hline$R_{u}(\mathrm{Kbps}), \beta=0$ & 960 & 480 & 240 & 60 & 15 & 15 & 15 & 60 & 15 & 60 \\
\hline$R_{u}(\mathrm{Kbps}), \beta=0.1$ & 960 & 480 & 240 & 60 & 15 & 15 & 15 & 60 & 15 & 60 \\
\hline$R_{u}(\mathrm{Kbps}), \beta=0.5$ & 960 & 240 & 120 & 15 & 15 & 15 & 15 & 60 & 15 & 60 \\
\hline$R_{u}(\mathrm{Kbps}), \beta=0.8$ & 480 & 240 & 30 & 15 & 15 & 15 & 15 & 60 & 15 & 60 \\
\hline
\end{tabular}

Table 1: Throughput vs Path Loss for a system using OVSF codes, for 10 users. Uncoded QPSK is assumed. The target SINR per bit is $\gamma=5(\approx 7 \mathrm{~dB})$, corresponding to a probability of a symbol error of $P_{b} \approx 5 \times 10^{-3}$. 
Input : $\mathbf{l}^{*}=\left[l_{1}^{*}, l_{2}^{*}, \ldots, l_{N}^{*}\right], \mathbf{L}=\left[L_{1}, L_{2}, \ldots, L_{N}\right], \mathbf{E}_{b}^{t}=\left[E_{1}^{t}, E_{2}^{t}, \ldots, E_{N}^{t}\right]$

Output : $\tilde{\mathbf{l}}^{*}=\left[\tilde{l}_{1}^{*}, \tilde{l}_{2}^{*}, \ldots, \tilde{l}_{N}^{*}\right]$

Initialization :

$n:=0 ; J_{0}:=0 ;$ for $u=1, \ldots, N, \tilde{l}_{u}^{*}:=l_{u}^{*} ; S:=\{2, \ldots, N\}$

Step 1 :

$U:=\left\{u \in S \mid l_{u}^{*}=\min _{u^{\prime} \in S} l_{u^{\prime}}^{*}\right\}$;

$u_{m}:=\arg \max _{u \in U} E_{u}^{t}$;

if $\tilde{l}_{u_{m}}^{*}=L_{u_{m}}$ then $S:=S-\left\{u_{m}\right\}$ and repeat Step 1;

Step 2

$n:=n+1$

$\tilde{l}_{u_{m}}^{*}:=\tilde{l}_{u_{m}}^{*}+1$

$\triangle_{n}:=2^{-\tilde{l}_{u m}^{*}}$

$J_{n}:=J_{n-1}+\triangle_{n}$;

Step 3: if $J_{n} \neq 2^{-l_{1}^{*}}$ goto Step 1;

Step $4: \tilde{l}_{1}^{*}:=l_{1}^{*}-1$;

end

Figure 3: Algorithm for Lemma 1

Input : $P, R_{s}, R_{0}, \aleph_{u}=\left\{n_{u}^{\prime}, n_{u}^{\prime}+1, \ldots, R_{0} / R_{s}\right\}, \mathbf{E}_{b}^{t}=\left[E_{1}^{t}, E_{2}^{t}, \ldots, E_{N}^{t}\right]$ (in increasing order) Output : $\mathbf{n}=\left[n_{1}, \ldots, n_{N}\right]$

Initialization : for $u=1, \ldots, N, n_{u}=n_{u}^{\prime}$

$$
\begin{aligned}
& P_{t}=\sum_{u=1}^{N} E_{u}^{t} R_{s} n_{u}^{\prime} \\
& n_{1}=\max \left\{n \in \aleph_{1} \mid n+\sum_{v \neq u} n_{v} \leq R_{0} / R_{S}, \quad\left(n-n_{1}^{\prime}\right) \leq \frac{P-P_{t}}{E_{1}^{t} R_{s}}\right\}
\end{aligned}
$$

end

Figure 4: Rate Assignment Algorithm for Multicode CDMA Systems

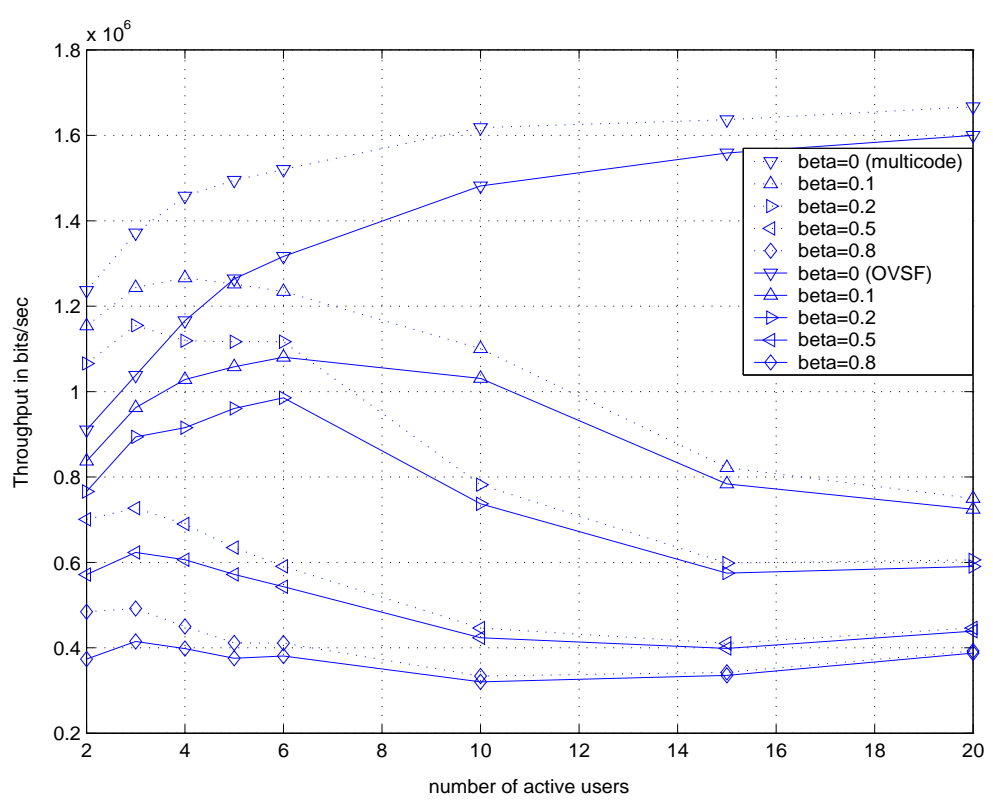

Figure 5: Comparison of the Average Throughput Results 


\begin{tabular}{|l|c|c|c|c|c|c|c|c|c|c|}
\hline User Index & 1 & 2 & 3 & 4 & 5 & 6 & 7 & 8 & 9 & 10 \\
\hline Path Loss $(\mathrm{dB})$ & 10 & 20 & 30 & 40 & 50 & 60 & 70 & 80 & 90 & 100 \\
\hline OF $(\beta)$ & 0.7 & 0.4 & 0.3 & 0.2 & 0.1 & 0.1 & 0.1 & 0.1 & 0.1 & 0.1 \\
\hline$R_{u, \min }(\mathrm{Kbps})$ & 15 & 15 & 15 & 15 & 15 & 15 & 15 & 15 & 15 & 15 \\
\hline$R_{u}(\mathrm{Kbps})$ & 15 & 15 & 15 & 15 & 960 & 480 & 240 & 120 & 30 & 30 \\
\hline
\end{tabular}

Table 2: Throughput vs Path Loss and $\beta$ for a constructed sample case. 